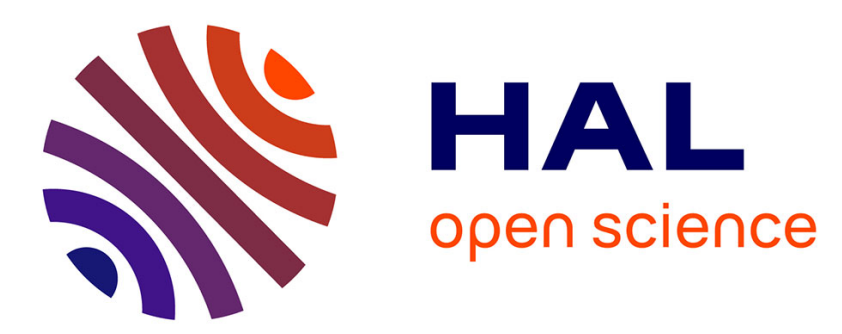

\title{
Des sportifs sans qualité ? Genèse du modèle étatique de production de l'élite sportive française
}

\author{
Sébastien Fleuriel, Manuel Schotté
}

\section{To cite this version:}

Sébastien Fleuriel, Manuel Schotté. Des sportifs sans qualité ? Genèse du modèle étatique de production de l'élite sportive française. Sociologie du Travail, 2015, 57 (4), pp.422-445. 10.1016/j.soctra.2015.09.007 . halshs-01227549

\section{HAL Id: halshs-01227549 \\ https://shs.hal.science/halshs-01227549}

Submitted on 28 Mar 2019

HAL is a multi-disciplinary open access archive for the deposit and dissemination of scientific research documents, whether they are published or not. The documents may come from teaching and research institutions in France or abroad, or from public or private research centers.
L'archive ouverte pluridisciplinaire HAL, est destinée au dépôt et à la diffusion de documents scientifiques de niveau recherche, publiés ou non, émanant des établissements d'enseignement et de recherche français ou étrangers, des laboratoires publics ou privés. 


\title{
Des sportifs sans qualité ? Genèse du modèle étatique de production de l'élite sportive française
}

\author{
Athletes Without Quality? Genesis of the State Model for \\ the Production of France's Sporting Elite
}

\author{
Sébastien Fleuriel et Manuel Schotté
}

\begin{abstract}
Résumé
Cet article s'intéresse au modèle français de production et de prise en charge de l'élite sportive en se focalisant sur la genèse du statut de sportif de haut niveau conçu comme une catégorie d'action publique apparue officiellement en 1975. II s'efforce de lier le contenu de la politique menée - sa forme et ce sur quoi elle débouche - avec le contexte dans lequel elle émerge et avec les propriétés des acteurs engagés dans sa production. C'est donc en revenant sur les con-ditions d'invention de ce statut que l'article rend compte de ce qui le caractérise. Deux dimen-sions centrales sont ainsi mises en évidence. Premièrement, le fait que ce statut ait été porté par une nouvelle génération de dirigeants avant tout soucieux de rénover l'organisation spor-tive en France conduit à ce que les sportifs en activité ne participent pas à son élaboration. Deuxièmement, le fait que ce statut ait pour visée de favoriser la performance et qu'il s'élabore dans un système de fortes contraintes nationales et internationales - qui interdisent aussi bien le recours au professionnalisme que le modèle des "sportifs d'État " - conduit à ce que ce statut soit défini en creux, par rejet de ce que ses promoteurs ne veulent pas. L'institutionnalisation du statut «par en haut » et «par défaut » explique que sa définition relève bien plus du souhait de permettre aux sportifs de s'adonner pleinement à la pratique que de la volonté de leur fournir un cadre protecteur sur le plan social.
\end{abstract}

Mots clés : Sport de haut niveau ; Statut ; Action publique ; Genèse ; Dirigeants.

\section{Abstract}

This article explores the French model for the production and management of the sporting elite, focusing on the genesis of the status of high-level athlete conceived as a category of public policy that officially appeared in 1975. It attempts to link the content of the policy - its form and outcome - with the context in which it emerges and with the properties of the actors involved in its production. The article thus identifies what characterises this status by returning to the conditions under which it was invented. Two central dimensions are highlighted. First, the fact that this status was backed by a new generation of managers primarily interested in renovating the organisation of sport in France, means that current athletes were not involved in its development. Secondly, the fact that the purpose of this status is to boost performance and that it was developed within a system of strong national and international constraint - which prohibited both professionalism and the "state athlete" model - led to this status being defined in negative terms, by the rejection of what its supporters wished to avoid. The "top-down" and "by default" way in which the status was institutionalised explains that its defini-tion has much more to do with the desire to enable athletes to dedicate themselves fully to practice, than with the wish to give them a protective social framework.

Keywords: High-Level Sport; Status; Public Policy; Genesis; Managers.

De nombreux travaux ont permis de mettre en évidence que les modes de production et de prise en charge des sportifs d'excellence sont très variables suivant les pays (Conzelmann et Nagel, 2003 ; Andersen et Ronglan, 2012 ; Hong, 2012). Partant de ce constat, cet article s'intéresse au modèle français de construction de l'élite sportive, qui 
s'avère très singulier dans le paysage international (Faure et Suaud, 2004 ; Henry, 2013). Les sportifs d'excellence français jouissent en effet d'un traitement particulier de la part de l'État. Ils bénéficient du statut de « sportif de haut niveau » 1 qui leur ouvre droit à un ensemble d'aides et d'aménagements censé tout à la fois leur permettre de s'entraîner de façon optimale, garantir leurs conditions sociales d'existence durant la carrière sporti-ve et leur ouvrir la voie à une reconversion réussie à l'issue de celle-ci. À cette fin, l'administration en charge des sports - ministère ou secrétariat d'État à la Jeunesse et aux sports (MJS et SEJS dans la suite du texte) suivant les moments publie régu-lièrement, sur proposition des fédérations sportives, une liste à quotas des ayants droit. Les " sportifs de haut niveau », au nombre de 7028 en 20122, se différencient des spor-tifs professionnels salariés tels qu'ils existent dans des disciplines comme le football (Rasera, 2013) et le cyclisme (Lefèvre, 2007), et des sportifs entrepreneurs de leur propre carrière que l'on retrouve dans des pratiques où la rémunération se fait à la prime comme le tennis (Waser, 1998) et l'athlétisme (Schotté, 2012), en ce qu'ils sont « assistés par l'État » (Fleuriel et Schotté, 2013).

Afin de rendre compte de cette situation particulière, l'article se centre sur la genèse du statut de sportif de haut niveau, conçu comme une catégorie d'intervention publi-que. Comprendre véritablement un problème social suppose en effet de revenir sur le processus qui a conduit à le construire comme tel et qui a abouti à la prime définition de la " réponse » apportée (Gusfield, 2009). C'est donc en s'intéressant aux conditions d'invention du statut de sportif de haut niveau que l'on s'efforce de rendre compte de ce qui le caractérise. Cet intérêt pour les «temps constituants 》 (Meimon, 2010) se jus-tifie dans la mesure où la première formalisation du statut, telle qu'elle apparaît dans la loi sur le sport de 1975, constitue la trame à partir de laquelle toutes les évolutions ultérieures du statut ont été envisagées.

Du fait du caractère instituant de ce cadrage initial, il importe de revenir précisément sur ce qui a sous-tendu son émergence et sa première formulation. Pour cela, le propos s'appuie sur un travail par archives (celles de l'administration de la Jeunesse et des sports, celles de divers groupes d'intérêt sportifs, auxquelles s'ajoutent quelques archi-ves privées), sur un dépouillement de la presse sportive et des bulletins de six fédéra-tions nationales ${ }^{3}$, sur des investigations auprès d'acteurs de l'époque (questionnaires auprès des sélectionnés olympiques de 1972 et campagne d'entretiens avec des diri-geants et des sportifs de la période) et enfin sur une analyse des débats parlementaires. II s'agit, par cette manière de procéder, de rendre compte aussi bien de la diversité des acteurs impliqués dans l'émergence de la politique publique considérée (Hassenteufel, 1995 ; Eymeri, 2003) que de la complexité des investissements et affrontements qui y président. Le propos s'inscrit donc dans une perspective de socio-histoire du politique visant à rendre compte des dynamiques de construction d'une catégorie de l'action pu-blique en décrivant précisément les configurations et les luttes qui la sous-tendent (Dubois, 1999 ; Noiriel, 2006 ; Buton et Mariot, 2009). Loin d'être enfermé dans le passé, ce « détour historique " se veut ancré dans une compréhension du présent qui en découle.

\footnotetext{
1 C'est du moins sous ce terme que l'usage désigne ce groupe. En réalité, l'article 16 de la loi $n^{\circ} 75-988$ du 29 octobre 1975 sur le sport, dite loi Mazeaud, fait référence à « la qualité d'athlète de haut niveau [qui] est déterminée par la fédération habilitée par le ministre chargé des sports ». Nous reviendrons sur cette différence dans la suite du texte.

2 Chiffres-Clés du sport, février 2013 : http://www.sports.gouv.fr/organisation/publication-chiffres-cles/Statistiques/Chiffres-cles/ (consulté le 19 mars 2014).

${ }^{3}$ Les fédérations françaises d'athlétisme, de basket-ball, de handball, d'escrime, de lutte et de ski. Elles ont été choisies en raison de la diversité des profils sociologiques de leurs adhérents et de leur positionnement sur le sort à réserver aux meilleurs d'entre eux. La consultation a porté sur la période 19691975.
} 
Nous cherchons à comprendre, au travers de la description de ce " premier geste de l'institutionnalisation » (Bezes et Pierru, 2012, p. 73), ce qui caractérise cette dernière, et tentons ce faisant de mettre au jour la spécificité du dispositif français d'encadre-ment des sportifs d'excellence qui perdure jusqu'aujourd'hui. Dans ce cadre, l'optique adoptée consiste à essayer d'établir une correspondance entre le contenu de la politique menée (sa forme et ce sur quoi elle débouche) et la structure relationnelle des acteurs qui ont été engagés dans son invention (Dubois, 2014). Comme cela a déjà été mis en évidence par d'autres travaux, le profil de ceux qui portent un problème a des effets sur son traitement (Bourdieu, 2000 ; Laurens, 2009). II importe donc de repérer, d'une part, quels sont les acteurs qui ont joué un rôle décisif dans l'élaboration de la politique publi-que considérée (Lagroye et al., 2006, pp. 512-513), et d'identifier, d'autre part, dans quel tissu de contraintes celle-ci a émergé, afin de rendre compte de ce à quoi elle a conduit.

\section{L'émergence d'une action publique pour le sport de performance}

La reconnaissance de la qualité de sportif de haut niveau apparue en 1975 dans la loi dite "Mazeaud » n'est pas le produit d'une décision ponctuelle. Elle procède d'une genèse complexe mettant en jeu des dynamiques qui dépassent de loin le moment de leur cristallisation dans une décision ${ }^{4}$. Cette disposition s'inscrit en effet à la croisée de plusieurs histoires au rang desquelles figure une série de réflexions et de mesures prises,

à compter des années 1950, pour favoriser l'émergence d'une élite sportive par des dirigeants du SEJS.

Ce ne sont pas là les premières formes d'intervention étatiques dans le sport. Mais jusqu'alors celles-ci avaient essentiellement visé la diffusion et le contrôle de la prati-que des exercices physiques pour le plus grand nombre à des fins éducatives et sani-taires (Clément et al., 1994 ; Callède, 2000). Si l'on peut parler d'une politique étati-que en direction du sport de performance à partir des années 1950, c'est du fait d'une succession de créations institutionnelles, dont le Bataillon de Joinville en 1956 (où sont affectés les appelés du contingent de niveau sportif international pour y bénéficier de conditions de préparation sans égales dans le monde civil), la création du corps des conseillers sportifs nationaux en 1958 (qui deviennent en 1966 les directeurs techniques nationaux : DTN), la mise en place de la «Délégation générale à la préparation olym-pique » (PO) en 1961, les sections sports-études en 1974 puis l'Institut national du sport et de l'éducation physique (INSEP) en 1975. Toutes ces mesures de soutien à la perfor-mance viennent ainsi en complément des premières dispositions en direction du sport éducatif, le lien se faisant par la rhétorique de la "locomotive ": le développement du "sport d'élite » est censé entraîner dans son sillage celui du « sport de masse ».

Ces mesures relatives à l'invention d'un dispositif français de prise en charge de l'élite s'inscrivent dans une conjoncture où différentes manières de considérer les sportifs d'ex-cellence entrent en concurrence au plan national : professionnalisme en vigueur dans le football (Wahl et Lanfranchi, 1995) et le cyclisme (Lefèvre, 2007) ; amateurisme strict ; modèle d'un sport d'État émergeant dans les pays de l'Europe de l'Est et qui trouve quelques résonances en France avec la mise en place de structures militaires d'en-cadrement ; valorisation de l'initiative privée et du pouvoir localisé des clubs (Loirand, 1996). C'est en réaction à cet ensemble, peu harmonisé et perçu comme inefficace (les résultats des sportifs français aux Jeux olympiques sont en baisse constante de 1948 à 1960), et à la faveur de la mise en œuvre d'une véritable administration du sport qui participe des visées planificatrices du régime gaullien (Le Noé, 2000), qu'interviennent les mesures étatiques en faveur du sport de haut niveau.

\footnotetext{
${ }^{4}$ Sur cette manière de problématiser les choses voir Politix, 2008.
} 
Comprendre l'intervention de l'État dans le domaine du sport suppose de renoncer à l'idée d'une confrontation binaire entre deux entités définies de façon abstraite : l'État et le Sport. Plutôt que de raisonner en termes d'intrusion d'un «bloc » (l'État) dans un domaine (le sport), il importe de mettre l'accent sur les luttes entre acteurs et sur les relations qui se tissent entre eux (Elias, 1991) pour donner forme au sport d'élite. II s'agit donc de rendre compte, comme le fait Vincent Dubois à propos de la politique culturelle (Dubois, 1999), des rapports qui s'établissent entre la fraction de l'espace étatique mobilisée pour le traitement public du sport de performance et les acteurs du «mouvement sportif ». Si certains aspects de ces relations entre État et acteurs fédéraux ont pu donner lieu à des oppositions ${ }^{5}$, les mesures visant à favoriser la préparation des sportifs d'élite ont toutes été accueillies favorablement par les dirigeants fédéraux : cela vaut pour la formation des entraîneurs, enjeu pour lequel « les fédérations trouvèrent auprès des pouvoirs publics un appui réel pour améliorer la qualité et le statut des meilleurs entraîneurs » dès la fin des années 1950 (Amar, 1987, p. 59), pour la mise à disposition par l'État de techniciens fonctionnaires qui a été reçue positivement par les dirigeants fédéraux (Honta et Juhle, 2013), et aussi, plus globalement, pour l'ensemble du dispositif d'aide à la préparation des sportifs mis en place.

Ces éléments étayent l'idée que l'action publique en faveur du sport de performance ne s'est pas faite en opposition avec les fédérations mais bien en s'appuyant sur elles. Cette convergence entre acteurs fédéraux et étatiques s'explique de façon croisée. Du côté des dirigeants fédéraux, un tel accord n'avait a priori rien d'évident, du fait notamment de leur profil : en 1962, la moyenne d'âge des présidents des fédérations de sports olympiques est de 61 ans, près de la moitié d'entre eux ( 9 sur 20) ayant au moins 65 ans $^{6}$. Autre caractéristique forte : ils sont seulement 6 à avoir été des sportifs de rang international (ou professionnel), et pour 4 d'entre eux la carrière de prati-quant s'est arrêtée au plus tard dans les années 1930, c'est-à-dire à un moment où le degré de préparation pour atteindre le niveau international était autrement moins exi-geant que dans les années 1960 (Erard, 2004). Éloignés aussi bien par leur âge qu'en termes de connaissance des exigences contemporaines du sport de compétition, ces dirigeants sont peu au fait des préoccupations des jeunes pratiquants aspirant à une carrière internationale. Ils sont aussi très majoritairement à distance du secteur public sur le plan professionnel dans la mesure où, sur les 19 présidents dont la profession est déclarée, 17 travaillent dans le privé et l'un des deux autres est commandant à la re-traite, devenu remisier en bourse des valeurs de Paris.

Comment comprendre, dans ces conditions, que ces dirigeants aient pu adhérer à un projet porté par les services de l'État et conduisant à mettre l'accent sur le sort réservé aux sportifs de haut rang ? Pour répondre à cette question, il faut rappeler que cet accord s'inscrit dans un contexte de forte critique - dans la presse notamment - de ces dirigeants fédéraux, considérés comme incapables de produire une élite performante et décrits comme « une caste de dirigeants pratiquement inamovibles ", à la tête de « véritables citadelles » qui « rendaient inévitable l'intervention de l'État, lequel se devait de corriger les injustices qui découlaient de cette situation ${ }^{7}$. Et comme les fédérations souffrent aussi d'un déficit structurel de moyens, on comprend que leurs présidents puissent, en dépit de leur probable réserve personnelle, avoir intérêt à s'appuyer sur les dispositifs étatiques pour former les élites de leur sport. L'enjeu central étant, pour eux, avant tout d'être réélus, tout laisse à penser qu'ils font preuve de pragmatisme à l'égard

\footnotetext{
${ }^{5}$ Plusieurs fédérations ont déposé un recours devant le Conseil d'État en 1962 à la suite d'un projet étatique de refonte de leurs statuts. À ce sujet, voir Loirand, 1996.

6 Calcul réalisé à partir des données figurant dans L'Équipe du 26 décembre 1962.

7 Gaston Meyer dans L'Équipe du 17 mars 1965.
} 
de tout ce qui peut renforcer leur reconnaissance dans leur discipline. En dépit de quelques critiques ponctuelles, ils donnent ainsi, de façon unanime, leur assentiment au pro-jet étatique dans la mesure où ils se voient offrir des moyens pour promouvoir leur élite sans que celle-ci leur échappe puisqu'ils restent maîtres d'œuvre des sélections des ath-lètes en équipe nationale ${ }^{8}$.

Du côté des acteurs étatiques, l'inclination à s'appuyer sur les fédérations pour cons-truire une politique nationale du sport de haut niveau a sans doute beaucoup à voir avec leur propre vécu : comme le montre Olivier Le Noé (2000), les principaux acteurs de cette politique ont été, pour la plupart, des sportifs ayant remporté des titres au niveau national ou international, ce qui n'a pu que forger en eux une propension à reconnaître

— et à se reconnaître dans - le modèle fédéral. Au-delà du coût politique qu'il y aurait eu, pour eux, à porter une véritable " étatisation du sport » déconnectée du monde fédéral, c'est aussi du fait de leur proximité réelle avec ce dernier que les acteurs du SEJS se sont adossés au " mouvement sportif » pour mener à bien la visée de promotion d'une élite sportive de rang international.

Le dispositif de production de l'élite se structure ainsi dans la rencontre des intérêts et caractéristiques des dirigeants fédéraux et des acteurs centraux du SEJS ; une rencontre qui, du même coup, tient partiellement les clubs à l'écart du processus de production des sportifs de haut rang.

\section{Une gestion personnalisée et autoritaire des sportifs}

Après avoir indiqué ce que sont les jalons institutionnels et les enjeux de l'action publi-que mise en œuvre en matière de sport de performance, il importe de décrire ce qu'elle induit pour les principaux concernés, à savoir les athlètes. Concernant les conditions de préparation, Jacques Defrance (1989) a montré, à propos de l'athlétisme, que la période des années 1960 se caractérise par une gestion autoritaire qui confère une faible autonomie aux sportifs. Loin d'être isolé, ce cas semble au contraire très significatif du mode de gestion impulsé : c'est en effet cette discipline qui est la première à se voir dotée d'un DTN et elle est ensuite prise pour référence. Le profil du premier DTN, Robert Bobin, est représentatif des caractéristiques de ceux qui sont nommés par la suite puisqu'il s'agit dans tous les cas de privilégier des « hommes de terrain ", reconnus pour leurs qualités de " meneurs d'hommes ». Ces DTN ont aussi en commun d'avoir environ quarante ans quand ils entrent en fonction, et d'avoir été avant cela militaires, enseignants d'éduca-tion physique et/ou sportifs de rang international ${ }^{9}$.

Ce type de profil est indissociable du fait que ces DTN ont tous été recrutés personnellement par Marceau Crespin. Fils de paysan devenu pupille de la nation, ce dernier s'engage tôt dans l'armée où les qualités qu'il manifeste au combat lui valent d'obtenir, sur la foi de ses faits d'armes sur le terrain, le rang de colonel. Devenant directeur de la PO, il y importe son « esprit commando » (Le Noé, 2000) et les méthodes qui ont fait son succès dans l'institution militaire. Le récit proposé, dans le livre Les commandos en France de Maja Destrem (1982), de sa manière de procéder quand il officiait encore dans l'armée, permet d'incarner la façon dont il dirigeait les soldats placés sous ses ordres :

\footnotetext{
8 Sauf pour les Jeux olympiques.

9 Cela s'appuie sur les informations fournies par O. Le Noé (2000) qui montre que les DTN sont des militaires (5), des enseignants d'éducation physique (4) ou des contractuels (11). Nos investigations complémentaires permettent de mettre en évidence que ces contractuels sont soit des éducateurs physiques ou moniteurs de ski (5), soit des anciens sportifs de haut rang (4), soit des cadres formés et/ou liés à l'armée (2).
} 
« [M. Crespin] ne lâche pas les hommes d'une semelle. Pendant des jours et des jours, il les prend par petits groupes et les entraîne. Entraîner, c'est peu dire. Placé sur un rocher qui domine le parcours, entouré de ses acolytes [...], il fait sauter des pétards pour habituer les hommes au bruit, leur tire dessus à la carabine américaine pour les aguerrir et les injurie sans vergogne, créant ainsi un climat qui les oblige à accepter et à vaincre les difficultés » (Destrem, 1982, pp. 76-77).

Même s'il est peu vraisemblable que M. Crespin ait procédé de la sorte avec les sportifs, tous les témoignages lus ou recueillis auprès d'athlètes de la période confirment sa proxi-mité avec eux, son exigence et sa valorisation de l'action virile. Si ces descriptions ne sont pas toujours dénuées d'ambitions hagiographiques, le fait que M. Crespin soit sys-tématiquement décrit comme un « fabuleux et infatigable meneur d'hommes » (Killy, 2000, p. 9) atteste d'un mode de gestion personnalisé et autoritaire, fondé sur des rela-tions "d'homme à homme ». Les propriétés personnelles de M. Crespin ont d'autant plus d'incidence sur le mode de prise en charge des sportifs émergeant dans les années 1960 qu'il se retrouve en situation d'inventer le rôle qui lui est confié dans une insti-tution naissante, la direction des Sports ${ }^{10}$, aussi peu dotée en personnel et ressources que faiblement bureaucratisée. Comme il a été montré par ailleurs, ceux qui investis-sent une institution en germe contribuent à lui donner forme dans la mesure où c'est à travers eux qu'elle se construit (Dubois, 1999 ; Meimon, 2010). En tant qu'acteur cen-tral dans la séquence de création institutionnelle de la direction des Sports, M. Crespin s'avère donc tout à fait décisif dans la forme qu'elle prend. Le fait qu'il soit en position de recruter les DTN qui vont être en charge de la préparation des sportifs renforce encore son importance, et ce d'autant plus qu'il sélectionne des profils qui lui res-semblent. Le pouvoir de M. Crespin - souvent décrit comme charismatique - est donc fonction des relais dont il dispose. II s'appuie ainsi sur la force de l'appareil d'État. Même balbutiant, le service auquel il appartient et qu'il contribue à mettre en forme lui confère en effet une légitimité ${ }^{11}$.

Si M. Crespin et les DTN qu'il recrute reconvertissent dans l'espace sportif des façons de faire acquises ailleurs, celles-ci ne peuvent être importées telles quelles auprès des athlètes. Elles se doivent d'épouser a minima les formes d'action qui prévalent dans cet espace. En l'occurrence, au regard de ce que sont les relations entraîneurs/entraînés depuis l'après-guerre, leur profil d'instructeurs, d'anciens sportifs ou d'enseignants d'éducation physique s'avère pleinement compatible avec les usages en vigueur qui se caractérisent par une valorisation du savoir pratique sur la connaissance théorique (Roger, 2003) et une conception du rapport entraîneur/entraîné comme une relation dissymé-trique entre celui qui sait et propose et celui qui exécute. Plus, on peut raisonnable-ment faire l'hypothèse que ces techniciens contribuent à durcir ce modèle, notamment sur le second aspect : autant l'importance d'un entraînement appuyé sur des connais-sances scientifiques devient progressivement un registre mobilisé par ces cadres - et notamment par M. Crespin qui s'adjoint les services d'un médecin à la direction des Sports, le Dr. Andrivet - , autant la question du rapport maître/élèves qui caractérise la relation entre l'entraîneur et " ses " athlètes ne semble jamais être remise en cause. Aucune archive ne fait en effet référence à un sportif acteur de sa préparation, de ses objectifs et des moyens d'y parvenir. Et aucune d'entre elles ne va dans le sens d'une telle implication du sportif dans son projet athlétique. Si tout est fait pour « leur accorder

\footnotetext{
${ }^{10}$ Nom générique par lequel on désigne le service — qui a changé plusieurs fois d'appellation — en charge du sport de performance.

${ }^{11}$ Il ne s'agit donc pas d'opposer pouvoir charismatique et pouvoir bureaucratique (Dobry, 2003), mais de souligner que le second contribue à rendre possible le premier, en mettant à la disposition de $\mathrm{M}$. Crespin un personnel disposé à se reconnaître dans ses actions, et en contribuant à leur donner une légitimité d'État.
} 
toutes facilités pour mener à bien leur entraînement $~^{12}$, la définition de ce dernier reste la prérogative exclusive du technicien. Les athlètes, souvent bien plus jeunes que ceux qui les encadrent, se retrouvent ainsi sans réelle maîtrise de la gestion de leur carrière et de leur préparation. Si un travail spécifique reste à mener sur ce qui conduit les sportifs à se fondre dans un tel fonctionnement, force est de constater qu'ils s'accommodent de la dépossession dont ils sont l'objet dans la mesure où ils ne remettent jamais en cause cette organisation.

II convient enfin d'ajouter une autre dimension du mode de prise en charge des sportifs, relative au régime des gratifications associées à la pratique. Si les règlements olympiques interdisent leur rémunération (voir infra), ces sportifs n'en sont pas moins aidés sur le plan financier. Mais cette aide reste très contrôlée et envisagée dans un re-gistre infantilisant. II est par exemple question de leur distribuer de l'« argent de poche ${ }^{13}$ pendant la période des compétitions. Et il est régulièrement signifié qu'il « paraît nocif au plus haut point de leur donner directement de l'argent : ils n'en sont pas du tout reconnaissants et y trouvent au contraire motif à contestation et à jalousie entre eux ${ }^{14}$. Outre le rapport à l'argent qui est engagé dans ces prises de position, ces dernières dé-notent aussi une conception très particulière des sportifs, qui s'accommode mal d'un rapport émancipé à la pratique.

\section{Un statut défini par en haut}

L'ensemble des mesures prises depuis les années 1960 en matière de sport de performance a pour but de fournir de meilleures conditions de préparation aux athlètes afin de leur permettre de faire face à l'accroissement de la concurrence sportive internatio-nale. Cette situation conduit à ce que l'investissement sportif devienne de plus en plus difficilement compatible avec une scolarité ou une vie professionnelle " normales ". Les acteurs du service de la direction des Sports du SEJS mobilisent alors une grande activité visant à permettre aux sportifs de se libérer pour s'entraîner et pour participer aux compétitions internationales. À la veille des jeux de Munich en 1972, faute d'un encadrement juridique et administratif spécifique, l'examen de la situation des présélectionnés olympiques au regard de leurs obligations scolaires ou professionnelles se fait au cas par cas. Cet examen appelle l'intervention régulière de l'administration cen-trale pour débloquer des situations particulières comme l'indique cette note de $\mathrm{M}$. Crespin au secrétaire d'État, relative à l'" assistance apportée aux athlètes ayant quelque chan-ce d'être qualifiés et dont le nombre dépasse évidemment de beaucoup ceux qui nous représenteront effectivement. Ainsi, chaque cas fut étudié individuellement ce qui eut pour effet de se traduire par des interventions multiples auprès des employeurs [...]. Ce travail, on s'en doute, a donné lieu à l'échange de milliers de lettres ${ }^{15}$.

C'est même parfois sous couvert du secrétaire d'État qu'une intervention est requise pour permettre de libérer certains athlètes, comme dans le cas suivant :

"Donc moi, à l'époque, j'étais champion de France, j'étais toujours un des leaders de ma catégorie, et ils me disent "on aimerait que tu sois dans les pré-sélectionnés". Et moi, je dis : "Je ne peux pas, moi. Je travaille. J'ai un métier, je ne peux pas". J'étais encore dans l'esprit "je ne peux pas perdre mon travail, ce n'est pas possible". [...] Alors j'étais

\footnotetext{
${ }^{12}$ Courrier de M. Crespin aux directeurs régionaux de la jeunesse, des sports et des loisirs, 12 juillet 1971 , AN/19780588/3. Toutes les cotes commençant par la mention «AN » signalent des documents consultés aux Archives nationales.

${ }^{13}$ Réunion de coordination préparatoire aux Jeux olympiques, 20 août 1972, AN/19780588/3.

${ }^{14}$ Note d'Yvan Ceas à l'attention de Marceau Crespin, 1972 (mois et jour non précisés), AN/19780588/4-5.

${ }^{15}$ Note rédigée par M. Crespin pour le secrétaire d'État en prévision de sa présentation au conseil des ministres le mercredi 23 août 1972, AN/19780588/4-5.
} 
contrarié, et c'est là qu'il s'est passé un événement un peu... qui était pour moi extraordinaire aussi. C'est que les... comment dire. Ma fédération en a fait part au ministre des sports à l'époque, qui était Herzog, qui a écrit au PDG [de mon entreprise].

[...] Parce qu'à l'époque toutes les compétitions internationales, championnats du monde, tout était sur mes congés. [...] Par contre ce qui est bien, c'est qu'ils m'ont demandé ce qu'il fallait faire pour... Donc ils m'ont libéré une semaine voire deux semaines par mois, en gardant mon salaire pour que j'aille suivre les stages de préparation " (Sélectionné olympique aux jeux de Munich de 1972, entretien du $1^{\mathrm{er}}$ décembre 2008).

Le courrier adressé à l'employeur le 5 juillet 1971 est ainsi libellé :

« Je me permets d'attirer votre bienveillante attention sur le cas de [Monsieur X], employé de votre entreprise. [Monsieur $\mathrm{X}$ ] est un de nos meilleurs spécialistes et, à ce titre, tout permet de penser qu'il sera sélectionné dans l'équipe qui représentera la France aux Jeux Olympiques de Munich en 1972. Toutefois, cette sélection ne pourra intervenir que dans la mesure où il dispose de conditions lui permettant de s'entraîner convenablement. Or, son affectation actuelle n'est pas favorable à sa préparation. C'est pourquoi, je vous serais très reconnaissant de bien vouloir étudier la possibilité de le faire bénéficier d'un poste [plus proche de son lieu d'entraînement]. Je m'autorise à vous formuler cette demande, certain que vous ressentirez ce que représente une sélection olympique pour un jeune athlète de valeur » (Archives privées remises par l'athlète concerné).

Le ton, relativement déférent, est symptomatique de la position de faiblesse de la puissance publique dans la constitution des sélections nationales. En la matière, rien ne semble aller de soi, et tout paraît négocié au fur et à mesure, ce qui traduit la réelle fragilité des dispositifs mis en œuvre dont l'efficacité semble dépendre étroitement, du moins dans une première phase, de l'intervention personnelle de leurs promoteurs. Bien en amont de la loi sur le sport de 1975, il apparaît donc qu'en incitant à l'élévation des performances sportives, l'administration centrale s'est progressivement trouvée face à un ensemble de questions d'une nature nouvelle, corrélative de l'intensification du temps effectivement consacré à la pratique. Face à l'exigence de préparation intensive, à l'impératif de libérer les sélectionnés aux moments des grandes échéances compétitives, les réponses de l'administration semblent dans un premier temps se faire au tout venant dans un concert de dispositions ajustées à mesure que les cas se présentent :

"À l'époque, le grand projet, c'était d'être à mi-chemin, la voie médiane, entre les pays de l'Est avec des athlètes d'État, et les pays anglo-saxons qui laissaient les gens se débrouiller. [...] Avec Crespin, c'était du coup par coup, il se débrouillait pour faire embaucher untel aux PTT, untel à la SNCF, c'était du... Parce qu'il était très proche des athlètes, Crespin, il les connaissait pratiquement tous et il savait quels étaient leurs besoins, mais ce n'était pas officialisé, c'était un peu... comment dire, artisanal » (Jacques Grospeillet, entretien du 27 février 2014).

Ce bricolage administratif - qui concerne aussi d'autres dimensions, notamment celle de la protection sociale des sportifs qui n'est pas toujours assurée ${ }^{16}$ - est coordonné par l'adjoint de Marceau Crespin, Jacques Grospeillet, qui est un ancien fonctionnaire de la France d'Outre-mer rapatrié en métropole au début des années 1960, et passé par l'École nationale de la France d'outre-mer (ENFOM). II est ainsi doté d'un profil sem-blable à celui des premiers administrateurs du ministère de la Culture, issus des mêmes filières et définis par Vincent Dubois comme des "fonctionnaires aguerris à des mœurs très peu administratives », qui valorisent la " souplesse », l'« inventivité » et le « prag-matisme » (Dubois, 1999, p. 177).

\footnotetext{
${ }^{16}$ Courrier de J. Grospeillet aux présidents de fédérations, 29 février 1972, AN/19780588/3.
} 
Tout concourt ainsi à ce que l'action de la direction des Sports à destination des sportifs de performance soit largement improvisée. Ces « errements inventifs » des « acteurs placés au cœur du dispositif étatique » (Offerlé, 1997, p. 3) importent, car ils contri-buent à définir un mode d'action et à faire émerger un problème qui n'existait pas jus-qu'alors, à savoir celui du statut des sportifs. Le mode de fonctionnement mis en place au cours des années 1960 commence en effet à faire l'objet de critiques au début des années 1970, notamment dans un rapport intitulé Sport de haute compétition et produit conjointement par le SEJS et le Comité national olympique et sportif français (CNOSF) qui vient d'être créé. II y est écrit que "l'importance du problème [celui de la produc-tion d'une élite performante] condamne désormais la politique du "coup par coup" "17. Ce propos s'inscrit dans une réflexion d'ensemble visant à réformer le sport français et fait suite à une " constatation irréfutable » : la poursuite d'une carrière à haut niveau « est incompatible avec les exigences dictées par la poursuite normale des études, par l'exer-cice d'une profession, ou par la vie actuelle du moins dans les structures françaises actuelles ${ }^{18}$. C'est en réponse à ce cadrage de la situation que le groupe de travail qui réunit des dirigeants du CNOSF et du SEJS propose la mise en place d'un statut pour les sportifs de haut rang.

Si l'idée de créer un tel statut n'est pas nouvelle ${ }^{19}$, la conjoncture du début des années 1970 lui donne une réelle consistance. L'importance alors conférée à cet enjeu a partie liée avec le profil de ceux qui le portent et qui ce faisant se l'arrogent. Les archives et une série de témoignages convergents ${ }^{20}$ mettent clairement en évidence le rôle central qu'ont joué Marceau Crespin et Jacques Grospeillet pour le SJES d'une part, et Claude Collard, Alain Danet et, dans une moindre mesure, Nelson Paillou pour le CNOSF d'autre part. Ces derniers ont en commun d'avoir été présidents de leur fédération nationale très jeunes (ils avaient respectivement 37,29 et 40 ans à leur accès à cette fonction) et d'avoir été, avant cela, investis dans une pratique de tout premier plan (en tant que compétiteurs pour les deux premiers, en tant qu'arbitre pour le dernier), ce qui les distingue très notoirement de leurs homologues. Ces « nouveaux entrants » se posent en rénovateurs du sport français et sont, avec l'appui souterrain de M. Crespin et J. Grospeillet, à la base de la création du CNOSF en 1972. Cette organisation, née de la fusion du Comité olympique français (COF) et du Comité national des sports (CNS), entend porter la voix du « mouvement sportif » et devenir l'interlocuteur central des pouvoirs publics en matière de structuration des affaires sportives. Au-delà des liens d'affinités personnelles qui unissent M. Crespin, J. Grospeillet, C. Collard et A. Danet ${ }^{21}$, et qui vont de pair avec un commun rejet, socialement ancré, d'autres dirigeants sportifs, comme le Comte Jean de Beaumont qui était le président du COF jusqu'en 1972, ou encore les présidents de fédérations décrits par C. Collard comme " de vieux dirigeants braqués ${ }^{22}$, cette convergence de vue résulte aussi d'une congruence d'intérêts. Tandis que le soutien, même discret, du SEJS est un appui pour C. Collard et A. Danet, la vo-lonté de ces derniers de dynamiser le sport français est accueillie très favorablement par M. Crespin et son adjoint qui y voient un possible relais de leur action.

\footnotetext{
${ }_{17}^{17}$ Rapport « Sport de haute compétition », CNOSF/SEJS, 1973, AN/19780587/17, souligné dans le texte.

${ }^{18}$ Ibidem.

${ }^{19}$ Elle est déjà mentionnée dans le rapport Lanet en 1954 (voir à ce sujet Loirand, 1996), dans l'Essai de doctrine du sport du Haut comité des sports en 1965, et évoquée à l'occasion par des journalistes, comme Gaston Meyer qui écrit dans L'Équipe du 26 septembre 1967 : « un problème devrait être, à notre avis, résolu d'urgence : une définition claire et un statut de l'athlète représentatif de ses droits présents et futurs et de ses devoirs ".

${ }^{20}$ Ceux que nous avons pu recueillir et ceux consignés par Yoan Grosset (2005).

${ }^{21}$ Le premier est le parrain de la fille du second. Et tous les quatre se recevaient mutuellement chez les uns et les autres. Ces données sont issues de l'entretien réalisé avec J. Grospeillet et du travail de Y. Grosset (2005).

${ }^{22}$ C. Collard, entretien réalisé et cité par Y. Grosset (2005, p. 45).
} 
C'est forts de cette alliance objective et de la création institutionnelle qui en découle (le CNOSF) qu'ils investissent l'enjeu du statut des sportifs de haut rang. Les actions conjointes qu'ils mènent avec la collaboration de $\mathrm{N}$. Paillou (réunion des fédérations olympiques qu'ils supervisent en octobre 1972 à I'INS, organisation d'un colloque à Chatenay-Malabry en juin 1972 puis d'un autre à Vichy en juin 1973, création d'une commission mixte pour la préparation olympique et la promotion des athlètes en 1973) peuvent être interprétées comme relevant d'une stratégie d'accaparement de la ques-tion. S'ériger en agents nécessaires d'une réforme décrite comme nécessaire (Laurens, 2009) est une façon pour eux de s'imposer comme les organisateurs sportifs centraux à l'échelle nationale, et ce contre d'autres acteurs qui défendent d'autres modes de prise en charge du sport d'élite comme, par exemple, ceux qui prévalent en football et en cy-clisme professionnels. En revendiquant la propriété du sport d'élite, il s'agit bien pour eux de se faire valoir comme les grands ordonnateurs du sport français.

Cette conquête du contrôle du sport de performance s'appuie sur une stratégie qui passe par une critique des dispositifs et façons de faire en vigueur, comme lorsque $\mathrm{M}$. Crespin écrit au secrétaire d'État que :

«Préparer les Jeux Olympiques n'est pas une entreprise aisée. Elle l'est d'autant moins que les organismes qui devraient normalement en avoir la charge ne sont pas à même d'assumer la tâche spécifique qu'elle implique. Les Fédérations sont mal armées pour effectuer un travail méthodique de tous les instants » (Note de M. Crespin au Ministre en vue du Conseil des ministres du mercredi 23 août 1972, AN/19780558/4-5).

La consultation des bulletins de six fédérations confirme cet état de fait : soit la ques-tion des conditions de préparation des sportifs de haut rang n'y est pas (fédérations d'escrime et de lutte) ou très peu évoquée (fédérations d'athlétisme et de ski), signe qu'elle n'est pas une question centrale pour les dirigeants en place ${ }^{23}$, soit elle fait l'ob-jet de prises de position régulières (fédérations de basket-ball et de handball) souli-gnant qu'il y a là un grave problème. Ceci indique, dans les deux cas, que les fédéra-tions ne sont pas en mesure de prendre ce problème en charge : au désintérêt des uns correspond en effet le sentiment d'impuissance des autres, qui considèrent qu'il y a là « un problème politique qui dépasse [...] largement la compétence des Fédérations ${ }^{24}$.

Ce n'est sans doute pas un hasard si les présidents les plus impliqués et les plus revendicatifs sont, pour cet enjeu, Nelson Paillou (handball) et Robert Busnel (basket-ball) et si tous les deux en appellent au soutien des pouvoirs publics : ils ont, à la différence de leurs confrères des autres fédérations, en commun d'avoir côtoyé le niveau inter-national en tant que pratiquants. Ils partagent aussi une autre propriété très distinctive chez les présidents de fédérations, à savoir leur proximité avec les services de l'État: N. Paillou est pupille de la nation et a fait toute sa carrière dans la fonction publique (agrégé de lettres, puis chargé de mission auprès du ministère de l'Éducation natio-nale), tandis que R. Busnel a été recruté comme DTN par M. Crespin après avoir été joueur puis entraîneur de l'équipe de France de basket-ball. On comprend, dans ces conditions, qu'ils soient tous les deux enclins à soutenir le mode de prise en charge des sportifs que dessinent les actions entreprises par les dirigeants du CNOSF et du SEJS.

\footnotetext{
${ }^{23}$ Cette assertion mérite quelques nuances. D'une part, parce que l'absence de publicisation d'un problème ne signifie pas forcément qu'il n'existe pas. Et d'autre part, parce que l'acuité de l'enjeu est variable sui-vant les conditions dont bénéficient les sportifs dans telle ou telle fédération. Le fait que de nombreux skieurs de l'équipe de France soient douaniers et bénéficient de facilités pour s'entraîner peut ainsi expli-quer que les dirigeants de la fédération de ski se sentent peu concernés par la question des actions à me-ner pour optimiser leurs conditions de préparation. Enfin, l'opposition évoquée ici, entre fédérations peu mobilisées et fédérations très impliquées sur la question des modalités permettant aux sportifs de se préparer, ne doit pas masquer leur commun rejet d'un autre mode de prise en charge des sportifs : le professionnalisme.

${ }^{24}$ Éditorial de N. Paillou dans le bulletin Handball ${ }^{\circ} 86,1972$.
} 
Ces derniers partagent en effet avec eux le constat d'un manque, et leurs propositions visent à y suppléer avec le concours de l'État. Cette présentation en termes de lacune est tout sauf neutre : agiter le spectre de l'échec sportif si rien n'est fait participe d'une entreprise de scandalisation (Offerlé, 1994) menée par les dirigeants du CNOSF et du SEJS afin de renforcer leur assise sur le sport français. Cette entreprise passe par voie de presse, notamment quand Claude Collard annonce, au nom du CNOSF :

«L'aide de l'État a été diminuée de 30 \% par rapport à celle de 1968 et nous ne sommes pas en état de préparer Montréal [où doivent se tenir les Jeux olympiques en 1976] 》 (L'Équipe, 23 mai 1973).

Elle passe aussi par la voie politique quand $M$. Crespin réclame des moyens au conseil-ler technique du secrétaire d'État :

«Par une note en date du 7 mai 1973 vous avez bien voulu me demander des précisions sur le montant des crédits supplémentaires qui pourraient être mis à la disposition du groupe de techniciens de haut niveau et sur l'utilisation prioritaire qui en serait faite [...]. De nombreux DTN se sont plaints de n'avoir plus de budget pour préparer Montréal. C'est pour cette raison qu'un effort particulier doit être fait sur ce plan [...]. Ne rien faire cette année équivaudrait à renoncer à toute participation en 1976 car on ne peut admettre que nous envoyions à grands frais une délégation qui ne ferait que de la figuration, surtout au Québec " (Note de M. Crespin à l'attention de Jean-Louis Langlais, conseiller technique, 14 mai 1973, AN/19780587/18).

Après avoir promu une lecture effectuée sur le registre du manque, les dirigeants du SEJS et du CNOSF se posent en sauveurs, seuls à même de redresser la situation, à la condition qu'on leur confie des moyens supplémentaires. La suite de l'exposé ne laisse en effet planer aucun doute sur le type de solution envisagée pour redresser la situa-tion sportive du pays :

«Dans la conjoncture actuelle on ne peut pas [...] réaffecter [les moyens] aux fédérations, car cet argent à n'en point douter suivrait la même voie que les subventions déjà attribuées et ne serait pas utilisé en vraie préparation olympique telle que nous l'entendons. La meil-leure solution me paraît donc être de verser ces deux millions au CNOSF en précisant à cet organisme qu'il ne devra les utiliser que selon nos instructions [...] » (AN/19780587/18, op. cit.).

Les dirigeants du SEJS et du CNOSF revendiquent ainsi la position de régulateurs du sport de performance pour mieux le contrôler.

Tous les éléments évoqués montrent que la promotion du statut de sportif de haut niveau s'apparente à une invention " par en haut ". Même s'ils sont pour la plupart des anciens pratiquants de bon niveau, les positions sociales plutôt élevées des dirigeants du SEJS et du CNOSF ${ }^{25}$, ainsi que le saut générationnel que leur fonction de représen-tation induit, contribuent à les tenir à relative distance des conditions d'existence des sportifs en activité, lesquels s'expriment d'ailleurs peu sur le sujet. L'émergence du sta-tut ne doit rien, en effet, à une quelconque mobilisation impulsée par ces derniers. Comme le raconte un athlète de la période (sélectionné aux jeux de 1964 et 1968), ce n'était en rien une revendication des sportifs en activité :

"Moi, j'étais athlète, ça me passait très largement au-dessus de la tête puisque ce n'était pas ma préoccupation. J'étais dans la vision du sport plaisir, du sport éducatif, du sport loisir. Je n'étais pas du tout dans la vision du sport professionnel, même je récusais cette dimension. [... En 1968], il y avait déjà des discussions [sur le statut d'athlète de haut

\footnotetext{
${ }^{25}$ Outre ceux dont les situations professionnelles ont déjà été évoquées, il convient d'ajouter que C. Collard est ingénieur (Centrale Paris) puis chef d'entreprise, tandis qu'A. Danet a été avocat avant d'occuper des fonctions de direction dans la presse.
} 
niveau] mais ça ne nous arrivait pas, à notre niveau, pour nous demander notre avis ou autre chose de ce genre. Ça se passait très largement au-dessus de nos têtes, nous n'étions pas concernés par cette affaire-là » (Entretien du 24 janvier 2014).

II est d'ailleurs symptomatique que la Fédération des internationaux du sport français, créée le 6 décembre 1974 et dont l'un des objets est l'" assistance aux internationaux et anciens internationaux de tous sports momentanément déshérités ainsi qu'à leur famille ${ }^{26}$, n'investisse pas cette question et se concentre sur une activité mondaine qui conduira à l'organisation d'un événement annuel appelé « les Gloires du sport » ${ }^{27}$. II en va de même des divers groupements de sportifs comme le groupement des inter-nationaux d'athlétisme, fondé en 1945, ou celui des nageurs, créé en 1946, qui n'inter-viennent en aucun cas dans les débats relatifs au statut et à la condition des sportifs de haut rang. Le même constat s'impose également concernant les divers syndicats qui structurent l'espace sportif, à commencer par ceux qui représentent les professionnels de l'encadrement sportif que sont les moniteurs de ski ou de tennis. Nous n'avons en effet relevé aucune mention de ces derniers dans les diverses archives mobilisées.

Si l'on réfère ce constat aux conclusions des travaux relatifs à l'histoire sociale des professions, qui montrent que la reconnaissance d'un groupe professionnel passe le plus souvent par un intense travail corporatiste (Boltanski, 1982 ; Abbott, 1988 ; Karpik, 1995), on voit ce que le cas des sportifs a de singulier. Les travaux portant sur des univers pro-ches permettent de montrer une autre particularité, à savoir que, contrairement aux ar-tistes qui se sont construits autour du refus de toute tutelle étatique (Sapiro, 2006), les sportifs n'ont pas rejeté celle-ci, bien au contraire ${ }^{28}$. Cela ne signifie toutefois pas qu'il faille conclure à une quelconque allergie des sportifs français à la revendication - le cas du mouvement et de la grève menés à la même période par les footballeurs profes-sionnels prouve en effet qu'il n'en est rien. II convient plutôt de référer leur « passivité »

à la configuration particulière dans laquelle la genèse du statut se réalise : le fait qu'ils soient éclatés en différentes disciplines sportives, engagés dans la pratique sur un mode vocationnel et pris dans un mode de gestion qui leur confère une autonomie très limitée conduit à ce qu'ils restent extérieurs à ce qui se décide en leur nom. Et comme ils ne sont en aucun cas sollicités par les promoteurs du statut, on comprend qu'ils restent à la marge des réflexions relatives à la forme que celui-ci doit prendre.

\section{Un statut défini par défaut}

Bien qu'ancrée dans la spécificité de la configuration française, la genèse du statut d'ath-lète de haut niveau est indissociable de l'existence d'un acteur international, le Comité international olympique $(\mathrm{ClO})$. Pensée comme devant permettre une amélioration des performances des sportifs français aux Jeux olympiques, la forme donnée au statut d'athlète doit nécessairement tenir compte des règlements de l'institution dont ses promoteurs visent la reconnaissance. Or, ces règlements interdisent toute participation olympique aux sportifs étiquetés comme professionnels.

L'action des dirigeants du CNOSF et du SEJS est ainsi située dans la tension entre l'impératif de maintien des sportifs dans une pratique intensive et le risque d'un professionnalisme jugé inacceptable. Outre le cloisonnement qu'ils opèrent avec les débats qui traversent le football professionnel français à la même époque, ces dirigeants condamnent

\footnotetext{
${ }^{26}$ Journal Officiel, 5 janvier 1975.

${ }^{27}$ Archives nationales du monde du travail, 2009006/1-45.

${ }^{28}$ Alfred Wahl (1990) indique même que, lors d'un des seuls mouvements portés par des sportifs sur des ques-tions d'organisation de leur pratique, les protestataires faisaient appel à l'État pour assurer l'autonomie de leur espace de pratique, et ceci durant Mai 1968 !
} 
fermement les sportifs qui se rendent coupables de tirer des rémunérations de leur pratique, comme l'illustre le cas de la radiation de skieurs au tournant des années 1970 $\left(\right.$ Faure, 2010) ${ }^{29}$. Ils s'efforcent alors de construire un modèle de prise en charge qui permette une spécialisation athlétique sans pour autant encourager un professionnalisme proscrit par le $\mathrm{ClO}$ et érigé en repoussoir. Pour trouver une solution acceptable, les promoteurs d'un modèle français multiplient les observations et comparaisons internationales : une note datant de 1975 fait état des réflexions relatives à l'observation des modèles japonais, italien, tchécoslovaque et australien ${ }^{30}$, et une série de voyages d'études est menée, particulièrement en URSS (août 1973) et en Chine (décembre 1973- janvier 1974) ${ }^{31}$. L'émergence du modèle français ne procède donc pas d'une logique insulaire mais s'inscrit dans une dynamique plus générale de circulation des savoirs et des techniques en matière de production de performance, d'autant que la question du statut à donner aux sportifs n'est pas propre à la France. Elle est discutée à la même époque dans d'autres pays, comme par exemple les ÉtatsUnis (Hunt, 2007 ; Turrini, 2010).

On ne saurait décrire la création du statut de sportif de haut niveau comme la stricte réponse aux conditions d'admission aux Jeux olympiques : les dirigeants français n'ont cessé de jouer avec la règle, comme le dénote une lettre adressée au COF par le CIO en 1972 qui s'inquiète de l'existence d'« un système d'aide matérielle [...] offerte aux meilleurs athlètes de votre pays, afin de "faciliter" et d' "encourager" leur préparation ${ }^{32}$, ce qui s'avère alors contraire aux règlements olympiques et qui est pourtant en vigueur en France depuis les années 1960. II est d'ailleurs parfois fait explicitement référence à cette possibilité de jeu avec la règle, entendue comme " un amateurisme olympique bien compris ${ }^{33}$. Force est toutefois de constater que l'assouplissement des règles internationales définissant l'amateurisme, qu'entérine le Congrès olympique de Varna en 1973, offre une plus grande marge de liberté aux dirigeants français dans la mesure où le nouveau président du CIO, Lord Killanin, explique dans L'Équipe du 23 août 1973 :

«Si j'apprends que tel athlète s'est entraîné durant deux mois dans un endroit spécial, mais n'a pas reçu en contrepartie une somme d'argent supérieure à son traitement habituel dans sa profession, je ne songe pas à le disqualifier ».

Les aides matérielles aux sportifs deviennent alors possibles, sous forme de dédomma-gements. Bien que, dans un état antérieur (et plus contraignant) des règlements olym-piques, de tels soutiens financiers aient été proposés aux sportifs français, l'assouplis-sement des règles internationales ne donne pas lieu à un renforcement de cette prati-que. Diverses solutions d'aide sont certes envisagées, comme celle exprimée dans une note remise à M. Crespin en 1972 :

«Lorsque l'on veut aider un sportif il faudrait prendre contact avec son employeur et lui proposer d'accorder une sorte de crédit d'heures d'entraînement comparable au crédit d'heures syndicales. Ces heures seraient payées par le Secrétariat d'État à l'employeur, celui-ci versant donc son salaire complet à l'athlète. Ce système aurait l'avantage de permettre aux sportifs de haut niveau de poursuivre parallèlement une activité professionnelle " (Note d'Y. Ceas à l'attention de M. Crespin, 1972, mois et jour non précisés, AN/19780588/4-5).

\footnotetext{
${ }^{29}$ Dans les faits, il s'avère que les contournements de l'interdiction du gain étaient très fréquents, ce que les dirigeants sportifs ne pouvaient ignorer. Les sanctions tombaient quand ces gains prenaient une dimension publique.

${ }^{30}$ AN/19780587/3.

${ }^{31}$ AN/19780587/20.

${ }^{32}$ Lettre d'Arthur Takac, directeur technique du CIO, au COF, 13 avril 1972, AN/19780588/9.

${ }^{33}$ Rapport «Sport de haute compétition », CNOSF/SEJS, 1973, AN/19780587/17.
} 
Ce possible latéral ne connaît toutefois pas de prolongement. Il en va de même pour une autre piste un temps envisagée :
"À la solution qui consistait à apporter une aide financière directe aux sportifs désireux d'accéder au niveau des meilleurs, il est préféré une forme plus élaborée associant le plus souvent une prise en charge partielle et temporaire à une préparation réelle de l'avenir social » (Rapport sur la participation olympique aux JO de Munich, 1972, mois et jour non précisés, AN/19780588/4-5).

Le soutien logistique est finalement préféré aux aides financières directes, ce qui dénote une vision particulière de ce que doivent être les conditions d'existence d'un sportif. Si tout est fait pour faciliter sa préparation, il est refusé que le sport puisse être une activité à temps plein. C'est ce qu'exprime, entre autres documents, un rapport qui fait suite aux Jeux olympiques de Munich :

"S'il est indispensable de placer les athlètes dans des conditions qui leur permettent de se préparer, il n'est pas bon que l'entraînement soit leur seule occupation. II faut éviter qu'ils s'amollissent et leur demander beaucoup en compensation de ce qu'on leur offre " (Synthèse des réflexions des chefs de délégation à l'issue des JO de Munich, 1972, mois et jour non précisés, AN/19780588/4-5).

On retrouve la même vision dans un courrier, noté comme confidentiel et relatif aux aménagements de service à accorder aux fonctionnaires et salariés susceptibles de se qualifier aux Jeux olympiques, de M. Crespin :

« Je pense qu'il ne serait pas bon que la décharge de service soit complète en raison des incidences psychologiques que l'on peut prévoir " (Courrier de M. Crespin aux directeurs régionaux de la jeunesse, des sports et des loisirs, AN/19780588/3).

Les dirigeants qui commandent la mise en place du statut de sportif de haut niveau semblent ainsi habités par la hantise que la pratique exclusive du sport puisse détourner les athlètes de la " vraie vie ". Si cette vision n'est sans doute pas exempte d'une condescendance à l'égard de sportifs dont il est postulé qu'ils s'aviliraient en vivant dans une " bulle ", il importe de voir qu'elle est liée à une vision du sport comme devant être au service de la fabrication des personnalités, comme l'exprime, exemple entre mille, une circulaire interne du CNOSF :

"Le sport n'est pas un but, mais un moyen d'éducation, de formation permanente et son rôle est avant tout social » (Circulaire interne du CNOSF n³9, 26 décembre 1973).

Cet attachement au principe d'un sport formateur permet de nuancer empiriquement l'opposition consacrée entre " sport éducatif » et "sport de compétition », et ce pour au moins deux raisons : d'abord, du point de vue de l'argumentaire employé, on cons-tate, aussi bien chez les dirigeants fédéraux qu'à la direction du SEJS, une relation de complémentarité entre la démocratisation des pratiques et un sport de haut niveau conçu comme la "locomotive » de celle-ci, brouillant ainsi à l'envi les frontières entre les domaines censément clivés de la " masse » et de l'« élite ". Une deuxième raison tient aux profils des dirigeants et des pratiquants : si, comme on l'a vu plus haut, un grand nombre de techniciens est issu de l'enseignement de l'éducation physique et sportive (EPS), cela vaut aussi pour les pratiquants puisque, pour les jeux de Munich (1972), près d'un sélectionné français sur deux en situation d'exercer un emploi est professeur d'EPS ou assimilé (maître auxiliaire, etc.) ${ }^{34}$. Les acteurs de l'éducation physique se trou-vant euxmêmes en situation d'appartenir à l'élite sportive, c'est de fait l'opposition sup-

\footnotetext{
${ }^{34}$ Nous tirons ces données de notre enquête sur la « reconversion des sportifs de haut niveau » (Fleuriel et Schotté, 2011) développée à partir de la population des sélectionnés aux jeux de Munich : sur les 53 spor-tifs ayant déclaré être en situation d'emploi pendant la préparation aux jeux, 23 l'étaient dans le domai-ne de l'éducation physique.
} 
posée entre "sport éducatif » et « sport de compétition » qui se trouve contestée, l'existence même du second étant travaillée de l'intérieur par les impératifs du premier.

Ce n'est donc pas seulement en vertu des règlements olympiques que le professionnalisme est refusé. C'est aussi parce qu'accepter ce dernier reviendrait à reconnaître une coupure entre élite et masse et à récuser toute l'économie de pensée sur laquelle s'est construite la politique étatique en faveur du sport, qui conçoit les élites comme des modèles aspirant dans leur sillage un développement de la pratique sportive (Fleuriel, 2004). Le rejet du professionnalisme s'incarnant principalement contre une professionnalisation par le marché, l'idée d'une possible prise en charge des sportifs par l'État est évoquée. Cette piste sera toutefois rejetée, au motif suivant :

« Notre conception du sport récuse tout à la fois un sport d'État qui fait du champion un fonctionnaire et un sport soumis aux seuls intérêts privés. L'État doit donc se montrer respectueux de l'autonomie du mouvement sportif, tout en lui apportant son aide » ( $P$. Mazeaud, Assemblée nationale, deuxième séance du 29 octobre 1973, p. 4978).

Le sportif d'élite ne peut plus être un pratiquant qui s'adonne à sa discipline sur la seule base de la passion individuelle dans la mesure où cet amateurisme total n'apparaît plus ajusté aux exigences de la compétition internationale. II ne peut pas non plus être un professionnel, ni un « athlète d'État » comme l'indique une version de l'avant-projet de loi où il est affirmé :

"Ce "statut" doit permettre d'éviter à l'élite de tomber sous la coupole des intérêts commerciaux ou sous l'emprise de l'État, et de bien figurer dans les compétitions internationales et notamment olympiques »(Avant-projet $\mathrm{n}^{\circ} 2$, non daté, estimé à 1974, AN/19780587/1).

Les réflexions qui sous-tendent la création du statut d'athlète de haut niveau sont marquées par la rareté des discours affirmatifs et la récurrence de formes négatives, de telle sorte qu'il semble se définir par défaut. La détermination de la forme que doit prendre le statut se fait ainsi en creux, sur la base du rejet de ce que ses promoteurs ne veulent pas. Cette construction sur le mode de la soustraction conduit à ce que soit ins-tituée une formule minimaliste qui raisonne en termes d'aide. II s'agit de permettre aux sportifs, par le biais de soutiens matériels (conditions de préparation) et financiers (sous la forme de bourses), de se consacrer pleinement à leur pratique, sans pour autant les considérer comme des travailleurs à qui des droits sociaux seraient conférés.

\section{Appropriation et légitimation politiques}

La genèse du statut de sportif de haut niveau relève pour une large part d'un travail réa-lisé par l'administration centrale en relation avec le CNOSF. II n'accède pourtant à une définition officielle qu'avec la loi sur le sport de 1975 qui l'institue et le légitime. Ce passage par la voie législative était une demande du CNOSF, dont les dirigeants n'ont pas seulement cherché à peser sur le contenu de l'action publique en question mais aussi sur la forme qu'elle devait prendre. Comme l'exprime C. Collard en 1973 :

" Hormis les moyens financiers à mettre en œuvre, c'est autant, sinon plus sous l'angle législatif que ces problèmes doivent être examinés pour définir le statut de ces sportifs » (Exposé du président C. Collard, Assemblée générale du CNOSF, 24 mars 1973, AN/19780588/9, souligné dans le texte).

Le déplacement de la question vers la scène politique a partie liée avec l'arrivée de Pierre Mazeaud à la tête du SEJS en 1973. Comme le note Jean-Michel Eymeri (2003), la définition de ce qui est central dans la vie d'un ministère se renégocie à chaque nomination d'un nouveau ministre (ou secrétaire d'État). Les archives montrent en l'occur-rence que $P$. Mazeaud manifeste d'emblée un intérêt et une activité plus grands que 
ses deux prédécesseurs dans les dossiers relatifs au sport de performance. Alors que François Missoffe et Joseph Comiti étaient, de par leurs pratiques personnelles, large-ment extérieurs au sport de compétition, P. Mazeaud peut faire valoir sa connaissance et la reconnaissance internationale qu'il a acquises dans le monde de l'alpinisme. De plus, bien qu'âgé de seulement 44 ans au moment de sa nomination, il dispose déjà d'une solide expérience politique : député des Hauts de Seine depuis 1968, il a aussi été chargé de mission dans plusieurs ministères (dont celui de la Jeunesse et des sports dans les années 1960). Et sitôt nommé secrétaire d'État (en avril 1973), il indique vou-loir soumettre au Parlement une « loi d'orientation du sport français » visant à « défi-nir une politique sportive à long terme ${ }^{35}$. Même s'il est en conflit avec M. Crespin (qui sera remplacé en 1974), son projet s'inscrit dans la continuité de ce qui a été enga-gé avant son arrivée : il affirme au CNOSF " son désir de tenir compte du résultat de [leurs] recherches grâce à une coopération étroite avec [cette] Confédération ${ }^{36}$. Et dès sa première séance de travail avec les dirigeants du CNOSF et les principaux admi-nistrateurs de la direction des Sports du SEJS en juin 1973, il convient de l'importance centrale que revêt la question du statut de sportif de haut niveau :

«En raison de l'urgence du problème, il fut admis que le premier thème de réflexion porterait sur les problèmes posés par la préparation des athlètes de Haut Niveau [...]. II fut en outre décidé d'élaborer un "rapport politique" constitué en faveur de la promotion d'une politique sportive pour les athlètes de Haut Niveau » (Circulaire interne du CNOSF n²9, 13 juillet 1973, rédigée par N. Paillou, vice-président chargé de la commission «Sport de haut niveau et préparation olympique $»$ ).

Le fait que P. Mazeaud s'approprie l'enjeu du statut des sportifs ne signifie pas que les précédents «propriétaires du problème » (Gusfield, 2009) en soient dépossédés. En dépit de son opposition à $M$. Crespin, l'investissement de $P$. Mazeaud se réalise dans le prolongement de ce qui a été amorcé au préalable et que son « appropriation politique »(Bezes, 2009, p. 109) renforce en contribuant à sa légitimation. Ainsi la mission d' " élaborer un projet de statut de l'athlète de haute compétition » figure-t-elle dans le «projet d'un programme de travail pour l'année 1973-1974. Objectifs à atteindre au $1^{\mathrm{er}}$ juillet 1974 » que P. Mazeaud confie à ses services ${ }^{37}$.

Cette " mise en politique ", entendue comme la transformation d'une question en objet d'action publique (Barthe, 2006), qui s'est amorcée dans les années 1960 et que renforce l'action politique de P. Mazeaud, ne s'accompagne toutefois pas d'une politisation - au sens d'usages et d'affrontements partisans - de l'enjeu du statut de sportif de haut niveau. Ainsi, alors que tous les deux en parlent pendant leur campagne pour l'élection présidentielle, les propos de Valéry Giscard d'Estaing et de François Mitterrand ne se différencient pas sur ce point :

« Je tiens par ailleurs à la définition d'un véritable statut du sportif de haute compétition. La collectivité nationale doit aider à sa préparation, elle doit aussi l'aider à préparer sa réinsertion professionnelle et sociale " (V. Giscard d'Estaing, entretien accordé à L'Équipe, 10 juin 1974).

«Nous traduirons notre respect de la dignité du champion par sa préparation et son insertion dans la vie sociale. Un statut de sportif de haut niveau sera élaboré " (Conférence de presse de F. Mitterrand sur le sport, 14 mai 1974, AN/19789587/19).

L'analyse des débats parlementaires qui précèdent la promulgation de la loi de 1975 montre également que la question ne fait pas l'objet d'affrontements partisans. Cela s'ex-

\footnotetext{
${ }^{35}$ Courrier de N. Paillou et C. Collard aux présidents des fédérations, 6 juin 1973, AN/19780588/9.

${ }^{36}$ Ibidem.

${ }^{37}$ AN/19780587/19.
} 
plique notamment par le fait que, aussi bien sur le plan quantitatif (nombre d'occurrences) que qualitatif (hiérarchie des thèmes abordés), le sujet du statut des athlètes de haut niveau s'avère secondaire dans le cadre des discussions dont le projet de loi est l'objet (Martin, 1999). Ce dernier ne porte en effet pas uniquement, loin s'en faut, sur les sportifs d'élite. La question du sort réservé à ces derniers s'insère dans une réflexion dont l'objectif principal consiste à démocratiser la pratique sportive. Dans ce cadre, la question du statut des sportifs d'élite ne fait pas débat à l'Assemblée nationale tant qu'elle s'articule avec les registres du sport éducatif à travers sa fonction d'exemplarité pour la jeunesse qui demeure la préoccupation centrale du service ministériel dirigé par P. Mazeaud (Le Noé, 2000).

De plus, conformément à cet agencement institutionnel qui accole, dans l'intitulé même du secrétariat d'État, le sport à la jeunesse, la pratique sportive fait, à l'Assemblée, l'ob-jet d'une lecture qui insiste sur les vertus éducatives qui lui sont prêtées. Cette dimen-sion se repère dans l'argumentaire développé, qui affirme la promotion d'un sport ver-tueux, doté " [d'] un rôle formateur fondamental et [d'] une finalité : le dévelop-pement harmonieux de l'individu » ${ }^{38}$. La définition donnée du sport dans le rapport de la loi établi par le sénateur Roland Ruet (Républicains indépendants), au nom de la commission des affaires culturelles, est à ce titre évocatrice :

«Le sport, élément de culture, dont les motivations profondes ne peuvent se découvrir que dans une initiation précoce, intégrée à l'éducation, dès les premiers temps de la scolarité. Le sport, activité physique gratuite qui cesse d'être culture s'il se transforme en activité physique commerciale, qui cesse d'être, s'il ne manifeste pas la générosité physique, le don de soi qui en fait la noblesse, avec pour conséquence de réserver les deniers publics au sport éducatif » (R. Ruet, rapport au Sénat $n^{\circ} 350$ relatif au projet de loi sur le sport, Commission des affaires culturelles, 1975).

Citée en introduction du rapport réalisé par le député Henri Lavielle (Parti socialiste) à l'Assemblée nationale, cette définition indique quelle vision du sport est engagée dans le projet de loi présenté par P. Mazeaud ; il s'agit d'une vision largement consensuelle, si l'on en juge par les rapports parlementaires qui mentionnent des vagues d'applaudissements, émanant de tous bords, en écho aux envolées relatives aux vertus morales du sport ${ }^{39}$.

Tout se passe comme si ce partage d'une vision enchantée du sport neutralisait les oppositions, y compris syndicales ou émanant de groupes politiques attentifs aux conditions de travail des sportifs - et ceci alors que, par le passé, les communistes se sont montrés soucieux des droits de travail des sportifs (Amar, 1987). Cette faible politisation de l'action publique en matière de sport (Belorgey, 2011) ne doit bien sûr rien aux propriétés intrinsèques de ce domaine du social. Elle relève à l'inverse d'une entreprise constante et multiple de dépolitisation (Defrance, 2000) à laquelle participent notamment, mais pas exclusivement, les acteurs politiques. Le sport, en particulier de compétition, ne peut en effet constituer une ressource politique à haute valeur symbolique qu'à condition d'être au préalable "neutralisé " et présenté comme relevant de l'universel. L'investissement de cet objet par les parlementaires a donc pour condition première la mise à distance du politique : ce placement n'est en effet rentable en terme d'image que parce qu'il n'apparaît pas comme politique. On comprend, dans ces conditions, pourquoi la question du sport de haut niveau ne fait pas l'objet d'une lecture clivante sur les bancs de l'Assemblée. Le passage devant les députés du statut de sportif de haut niveau n'est pourtant pas sans incidence sur ce dernier : intégré dans une loi, il se trouve légitimé et inscrit dans le droit.

\footnotetext{
${ }^{38}$ Ernest Rickert, député UDR, Assemblée nationale, première séance du 2 octobre 1975, p. 6415.

${ }^{39}$ Notamment lorsque R. Ruet, rapporteur du projet de loi devant le Sénat, déclare : « Nous avons besoin du sport pour résister aux excès de la nôtre [de civilisation] » (Sénat, séance du 5 juin 1975, p. 1271).
} 


\section{La qualité VERSUS le statut}

Au regard de ce qui précède, il semble que, telle qu'elle a été pensée, la reconnaissance de la qualité de sportif de haut niveau relève bien plus d'un souci de permettre à des sportifs de s'adonner pleinement à leur pratique que d'une " préoccupation sociale ". Cela se décline de deux façons : lorsque des considérations relevant de ce registre sont évoquées, c'est d'abord sous l'angle du risque de se priver d'une partie de la population. Suite au constat que « beaucoup d'athlètes de qualité quittent prématuré-ment le sport $\gg$, il est affirmé que :

"La démocratisation du sport [...] doit encore s'accentuer [et que] l'élite sportive ne s'identifie plus comme cela a été le cas par le passé à une classe sociale privilégiée. II faut donc que le statut de l'athlète soit tel que l'accès à l'élite ne soit le fait que de la seule supériorité sportive et morale » (Exposé du président $C$. Collard à l'assemblée générale du CNOSF, 24 mars 1973, AN/19780588/9).

Ensuite, quand il est question de rémunérer les sportifs, la chose est toujours envisagée en suivant la logique du dédommagement et non celle du salaire. Le modèle mis en place relève ainsi d'une logique d'aménagement circonscrit, inscrite dans un registre moral. Le statut est en effet pensé en termes de devoirs réciproques et non en termes de contrat de travail. Cette représentation apparaît clairement dans une circulaire du CNOSF :

« Il est de notre devoir d'aider nos athlètes d'élite [...]. Si les dirigeants ont des devoirs, les athlètes eux aussi doivent comprendre que le soutien qui leur est apporté [...] implique de leur part des devoirs impératifs. Ils ont un contrat moral, non seulement avec leur Fédération, mais aussi avec leur pays et doivent avant tout considérer comme un honneur de le représenter » (Circulaire interne du CNOSF n 39, 26 décembre 1973).

En cadrant les choses sur le mode du don de soi et des gratifications symboliques, et en appelant à la morale de l'honneur, le modèle promu « implique [de la part des sportifs] l'acceptation de sacrifices familiaux, professionnels et financiers ${ }^{41}$.

Au bout du compte, ce n'est pas un statut auquel seraient associés des garanties sociales qui est promulgué. La loi de 1975 se contente de reconnaître une qualité de spor-tif de haut niveau, qualité dont la seule vertu est de donner droit à des aides et des amé-nagements $^{42}$. Le modèle qui se met en place en France durant les années 1960 et 1970 est donc, pour les sportifs d'excellence, celui d'un "sport aidé par l'État " (Chifflet, 2005). L'inachèvement du traitement réservé à ces derniers — dans la mesure où la qualité qui leur est reconnue ne leur ouvre droit à aucune protection sociale, si l'on excepte l'accès très tardif (2012) au régime général d'assurance vieillesse - est lié à la confi-guration particulière qui l'a vu apparaître. Son caractère partiel est en effet le produit des conditions de son institutionnalisation : un tel dispositif constitue, d'un côté, un moyen pour le SEJS d'agir dans le sens d'une élévation du niveau de performance des sportifs français sans pour autant se substituer complètement au « mouvement spor-tif »; de l'autre côté, cette formule minimaliste reçoit l'assentiment des fédérations, auxquelles sont offerts des moyens pour promouvoir leur élite sans que celle-ci leur échappe.

\footnotetext{
${ }^{40}$ Synthèse des réflexions des chefs de délégation à l'issue des JO de Munich, AN/19780558/4-5.

${ }^{41}$ Note de Max Gombert (DTN du cyclisme) aux coureurs ayant posé leur candidature à la sélection pour les JO, 24 février 1972, AN/19780588/3.

${ }^{42}$ L'article 16 de loi de 1975 est ainsi formulé : « L'État veille à garantir la promotion sociale des sportifs de haut niveau. Cette garantie prévoit notamment l'octroi d'aides diverses, d'aménagement et de réduc-tion des horaires de travail en fonction des impératifs d'entraînement et de compétition et des disposi-tions tendant à l'insertion ou à la réinsertion professionnelle ».
} 
Les sportifs se voient finalement proposer des conditions de pratique que l'on peut qualifier de "professionnelles " dans la mesure où tout est fait pour qu'ils puissent faire de l'entraînement leur activité principale. Mais, dans le même temps, cette activité n'est pas étiquetée comme relevant du domaine du travail. C'est alors toute la spécificité de la catégorie de sportif de haut niveau qui se révèle : cette forme stabilisée d'appréhension et de traitement des athlètes les envisage comme complètement différents des sportifs professionnels qui sont eux salariés et, à ce titre, bénéficiaires de droits sociaux. À la différence du sort réservé à ces derniers, la qualité d'athlète de haut niveau conduit à

\section{Encadré 1. La qualité de sportif de haut niveau depuis la loi de 1975}

L'adoption de la loi $n^{\circ} 75-988$ du 29 octobre 1975 relative au développement de l'éducation physique et du sport (loi Mazeaud) reconnaissant la qualité de sportif de haut niveau ouvre une sé-rie de dispositions légales et administratives qui en précisent et consolident progressivement le périmètre effectif. Si quelques tentatives de recensement des sportifs de haut niveau sont ini-tiées par les fédérations à partir de 1982, la première liste ministérielle des sportifs de haut ni-veau n'est officialisée qu'avec l'adoption de la loi n84-610 du 16 juillet 1984 relative à l'orga-nisation et à la promotion des activités physiques et sportives (loi Avice). Elle est définie à par-tir de quatre catégories, élite, senior, jeune, et reconversion, auxquelles s'ajouteront par la suite les juges et arbitres (loi n`92-652 du 13 juillet 1992 modifiant la loi $n^{\circ} 84-610$ du 16 juillet 1984 et portant diverses dispositions relatives à ces activités).

Les données disponibles sur les effectifs des sportifs de haut niveau des six fédérations évoquées dans notre développement (athlétisme, basket-ball, handball, escrime, lutte, ski) et sollicitées auprès du bureau des sports de haut niveau de l'administration centrale donnent une bonne idée du processus de définition post hoc d'une qualité sans véritable contenu au départ (voir le tableau 1). Ce service ne dispose en effet des informations qu'à compter de 1994 (à la suite de l'adoption le 3 mars 1993 de la Charte du sport de haut niveau par la Commission nationale du sport de haut niveau) et renvoie vers les fédérations pour les séries comprises entre 1984 et 1994. Nos démarches auprès de celles-ci pour compléter les données se sont toutes avérées infruc-tueuses au motif, par exemple, qu' « il nous est impossible de vous fournir ces renseignements car nous n'avions pas à l'époque le matériel informatique pour garder ces informations ; et nous n'avons pas d'archives assez anciennes sur le sujet » (correspondance du 16 février 2015).

L'évolution globale des effectifs (toutes catégories et toutes fédérations confondues) montre une hausse continue du nombre de sportifs de haut niveau jusqu'en 2009, date à partir de laquelle se formalise une politique de contention des effectifs cristallisée par la mise en place des par-cours d'excellence sportive dont l'objectif rend explicite le « resserrement du dispositif au profit de la qualité » pour se « recentrer sur le haut niveau international », de sorte que les « critères d'ins-cription sur les listes ministérielles haut niveau et espoirs seront revus dans le sens d'une rigueur accrue » (instruction $n^{\circ} 09-028 \mathrm{JS}$ relative à l'élaboration du parcours de l'excellence sportive du 19 février 2009). Les six fédérations concernées suivent globalement cette évolution en infléchissant leurs effectifs à partir de 2010 (voir la figure 1).

Parallèlement à ces dispositions qui touchent à la définition de la population concernée, la qualité de sportif de haut niveau s'est aussi progressivement enrichie d'un contenu social amen-dé au gré des circonstances, essentiellement à compter de la loi de 1984. Les principaux apports successifs sont les suivants : un aménagement des études scolaires et universitaires (loi $n^{\circ} 84-610$, articles 27 et 28) ; des mesures particulières pendant le service national (loi n`84-610, ar-ticle 30, abrogé depuis lors) ; des dispositions d'ordre socioprofessionnel (telles que l'accès à l'em-ploi public, les affectations préférentielles pour les fonctionnaires, l'aménagement des condi-tions de travail, les aides à l'insertion professionnelle, à la formation et à la recherche d'em-ploi ; loi n`84-610, articles 28, 29, 31 et 32 ) ; une surveillance médicale adaptée (décret $n^{\circ} 87-473$ du $1^{\text {er }}$ juillet 1987 puis loi $n^{\circ} 89-432$ du 28 juin 1989) ; un accès à des aides financières personnalisées (instruction n`95-012 JS du 16 janvier 1995), un régime d'avantages sociaux et fiscaux (arrêté du 27 juillet 1994 et loi du 13 juillet 1992 modifiant la loi du 16 juillet 1984) ; et enfin, depuis le $1^{\text {er }}$ janvier 2012, un accès au régime général d'assurance vieillesse (art. L.351-3 du Code de la sécurité sociale). 
une définition faiblement objectivée de la condition de sportif : une performance donne certes droit, un temps durant, à des conditions de préparation et à une série d'avantages. Mais ces possibilités offertes sont, d'une part, éphémères (elles ne durent que le temps où le sportif est jugé suffisamment compétitif), et, d'autre part, associées à très peu de garanties sociales (absence de sécurité sociale, d'assurance maladie, etc.).

Au regard de la brièveté et du caractère aléatoire des carrières sportives, on peut considérer que, tout en fournissant les conditions d'une préparation sportive de rang international, le dispositif ainsi créé officialise une forme de précarité assistée. La loi de 1975 n'est bien sûr que la version initiale, amendée par la suite, du traitement étati-que des sportifs de haut niveau (voir l'encadré 1$)^{43}$. Mais si un tel détour par la genèse de celle-ci se justifiait, c'est que cette loi pose les bases à l'aune desquelles les évolutions de la qualité de sportif de haut niveau seront envisagées par la suite.

Tableau 1. Évolution des effectifs des sportifs de haut niveau (catégories cumulées : élite, senior, jeune, reconversion)

\begin{tabular}{|l|r|r|r|r|r|r|}
\hline & 1994 & 1995 & 2000 & 2005 & 2010 & 2015 \\
\hline Fédération Française d'Athlétisme & 379 & 380 & 393 & 391 & 395 & 263 \\
\hline Fédération Française de Basket-ball & 132 & 121 & 158 & 185 & 240 & 190 \\
\hline Fédération Française de Handball & 139 & 110 & 115 & 116 & 173 & 173 \\
\hline Fédération Française d'Escrime & 110 & 120 & 132 & 101 & 144 & 143 \\
\hline Fédération Française de Lutte & 107 & 127 & 109 & 124 & 130 & 97 \\
\hline Fédération Française de Ski & 207 & 193 & 284 & 286 & 305 & 307 \\
\hline Toutes fédérations $(\mathrm{n}=61)$ & 5252 & 5683 & 5856 & 6161 & 7190 & 6581 \\
\hline
\end{tabular}

Source : Secrétariat d'État aux Sports / Bureau des sports de haut niveau, 2015.

Figure 1. Évolution (toutes catégories et toutes fédérations) des effectifs des sportifs de haut niveau depuis 1994

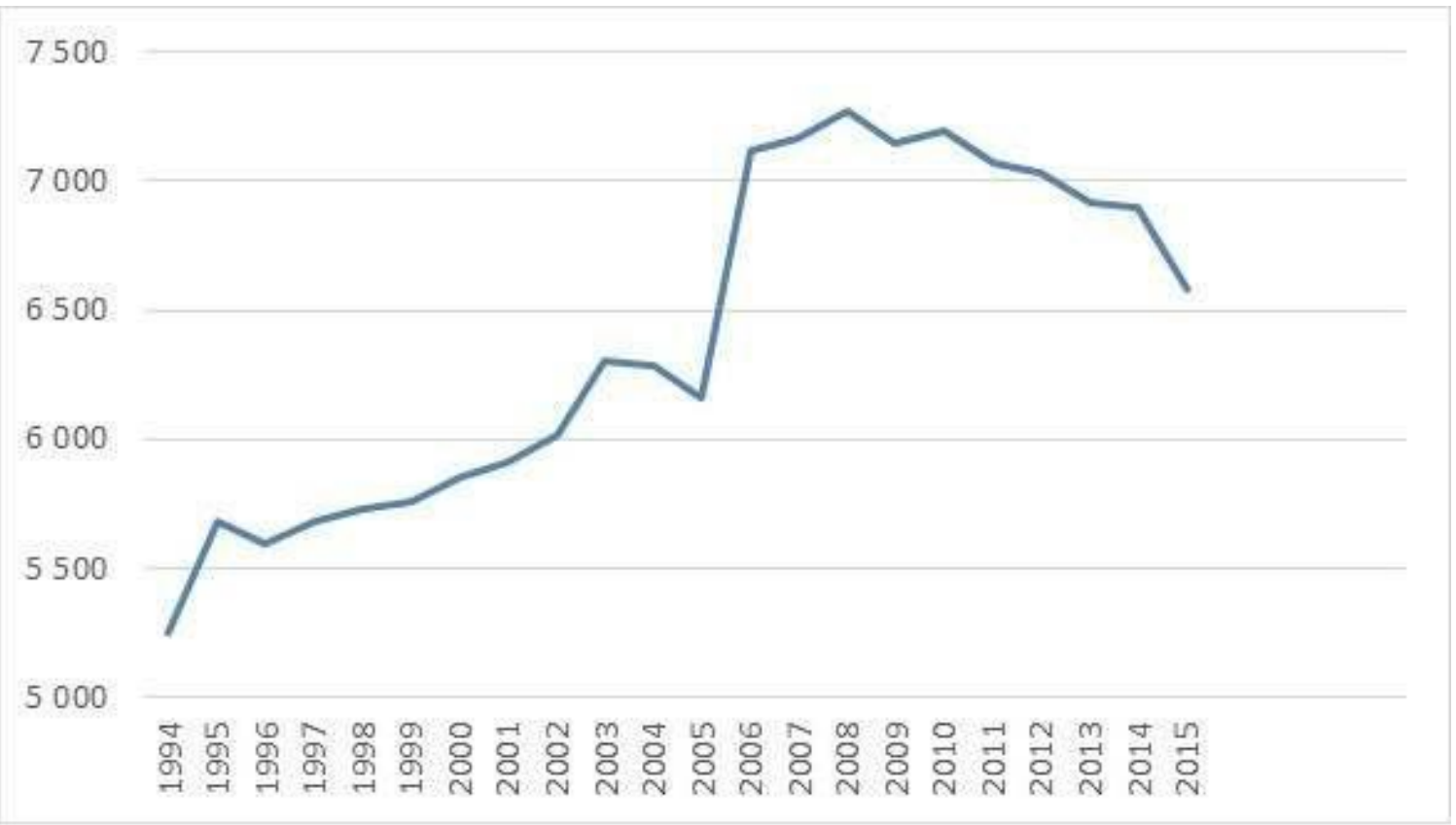

${ }^{43}$ II faudrait dire, en toute rigueur, que c'est la première version ayant force de loi. 


\section{Auteurs}

Sébastien Fleuriel

Centre lillois d'études et de recherches sociologiques et économiques (CLERSÉ),

UMR 8019 CNRS et Université de Lille 1

Bâtiment SH2, 59655 Villeneuve d'Ascq Cedex,

France sebastien.fleuriel[at]univ-lille1.fr

Manuel Schotté

(auteur correspondant)

Centre d'études et de recherches administratives, politiques et sociales

(CERAPS), UMR 8026 CNRS et Université de Lille 2

1, Place Déliot, BP 629, 59024 Lille Cedex,

France manuel.schotte[at]univ-lille2.fr

\section{Références}

Abbott, A., 1988. The System of Professions: An Essay on the Division of Expert Labor. The University of Chicago Press, Chicago.

Amar, M., 1987. Nés pour courir. Sport, pouvoirs et rébellions (1944-1958). Presses universitaires de Grenoble, Grenoble.

Andersen, S., Ronglan L., 2012. Nordic Elite Sport: Same Ambitions, Different Tracks. Copenhagen Business School Press, Copenhagen.

Barthe, Y., 2006. Le pouvoir d'indécision. La mise en politique des déchets nucléaires. Economica, Paris.

Belorgey, N., 2011. Les cadres de l'expertise publique. Les trois dimensions de l'expertise d'État saisies par une ethnographie des économistes de la Banque de France. Genèses 85, 93-114.

Bezes, P., 2009. Réinventer l'État. Les réformes de l'administration française (1962-2008). Presses universitaires de France, Paris.

Bezes, P., Pierru, F., 2012. État, administration et politiques publiques : les dé-liaisons dangereuses. La France au miroir des sciences sociales nord-américaines. Gouvernement et action publique 1 (2), 41-87.

Boltanski, L., 1982. Les Cadres. La formation d'un groupe social. Les Éditions de Minuit, Paris.

Bourdieu, P., 2000. Les structures sociales de l'économie. Le Seuil, Paris.

Buton, F., Mariot, N., 2009. Pratiques et méthodes de la socio-histoire. Presses universitaires de France, Paris.

Callède, J.-P., 2000. Les politiques sportives en France. Éléments de sociologie historique. Économica, Paris.

Chifflet, P., 2005. Idéologie sportive et service public en France. Mythe d'un système unifié. Presses universitaires de Grenoble, Grenoble.

Clément, J.-P., Defrance, J., Pociello, C., 1994. Sport et pouvoirs au xx ${ }^{\mathrm{e}}$ siècle. Presses universitaires de Grenoble, Grenoble.

Conzelmann, A., Nagel S., 2003. Professional Careers of the German Olympic Athletes. International Journal for the Sociology of Sport 38 (3), 259-280.

Defrance, J., 1989. Un schisme sportif. Clivages structurels, scissions et oppositions dans les sports athlétiques, 1960-1980. Actes de la recherche en sciences sociales 79, 76-91.

Defrance, J., 2000. La politique de l'apolitisme. Sur l'autonomisation du champ sportif. Politix $50,13-27$.

Destrem, M., 1982. Les Commandos de France. Les volontaires au béret bleu : 1944-1945. Fayard, Paris.

Dobry, M., 2003. Charisme et rationalité : le « phénomène nazi » dans l'histoire. In: Lagroye, J. (Ed.), La Politisation. Belin, Paris, pp. 301-323. 
S. Fleuriel, M. Schotté, « Des sportifs sans qualité ? Genèse du modèle étatique de production de l'élite sportive française »

Dubois, V., 1999. La politique culturelle. Genèse d'une catégorie d'intervention publique. Belin, Paris.

Dubois, V., 2014. L'État, l'action publique et la sociologie des champs. Revue suisse de science politique 20 (1), 25-30.

Elias, N., 1991. Qu'est-ce que la sociologie ? Éditions de l'Aube, Paris.

Erard, C., 2004. La dynamique socio-culturelle de l'élite athlétique française sous la IV ${ }^{\mathrm{e}}$ République : essai d'analyse prosopographique. Science et motricité 53, 105-118.

Eymeri, J.-M., 2003. Frontières ou marches ? De la contribution de la haute administration à la production du politique. In: Lagroye, J. (Ed.), La Politisation. Belin, Paris, pp. 47-77.

Faure, J.-M., 2010. Les skieurs de l'équipe de France (1968-1988). Reconversion et destins sociaux. In: Faure, J.-M., Fleuriel, S. (Eds), 2010. Excellences sportives. Économie d'un capital spécifique. Éditions du Croquant, Bellecombe-en-Bauges, pp. 201-249.

Faure, J.-M., Suaud, C., 2004. Pour une sociologie du sport réflexive. Les vertus du comparatisme. In: 3SLF (Ed.), Dispositions et pratiques sportives. L'Harmattan, Paris, pp. 379-392.

Fleuriel, S., 2004. Le sport de haut niveau en France. Sociologie d'une catégorie de pensée. Presses universitaires de Grenoble, Grenoble.

Fleuriel, S., Schotté, M., 2011. La reconversion paradoxale des sportifs français. Premiers enseignements d'une enquête sur les sélectionnés aux jeux olympiques de 1972 et 1992. Sciences sociales et sport 4, 115-140.

Fleuriel, S., Schotté, M., 2013. Le contractuel, l'entrepreneur, l'assisté : trois figures du travailleur sportif. Espaces Marx 33, 43-51.

Grosset Y., 2005. La création du CNOSF : la quête d'un nouveau pouvoir sportif (1965-1975). Mémoire de master recherche de l'université de Grenoble 1.

Gusfield, J., 2009. La Culture des problèmes publics. L'alcool au volant : la production d'un ordre symbolique. Economica, Paris.

Hassenteufel, P., 1995. Les groupes d'intérêt dans l'action publique : l'État en interaction. Pouvoirs 74, 155-168.

Henry, I., 2013. Athlete Development, Athlete Rights and Athlete Welfare: A European Union Perspective. The International Journal of the History of Sport 30 (4), 356-373.

Hong, E., 2012. Applying a Western-based Policy Community Framework to the Analysis of South Korean Elite Sport Policy: The Role of Businesses and Armed Forces. International Journal of Sport Policy and Politics 4 (1), 23-37.

Honta, M., Juhle, S., 2013. Les professions du secteur public saisies par la privatisation. Le cas des conseillers techniques sportifs. Gouvernement et action publique 2 (1), 63-87.

Hunt, T., 2007. Countering the Soviet Threat in the Olympic Medals Race: The Amateur Sports Acts of 1978 and American Athletics Policy Reform. The International Journal of the History of Sport 24 (6), 796-818.

Karpik, L., 1995. Les avocats entre l'État, le public et le marché, XIII ${ }^{\mathrm{e}}-\mathrm{xx} \mathrm{e}^{\mathrm{e}}$ siècle, Gallimard, Paris.

Killy, J.-C., 2000. Préface. In: Laget, S., Mazot, J.-P., Marceau Crespin, à la force de poignets. PPL, Chirac.

Lagroye, J., François, B., Sawicki, F., 2006. Sociologie politique. Presses de Sciences Po/Dalloz, Paris.

Laurens, S., 2009. Une politisation feutrée. Les hauts fonctionnaires et l'immigration en France (1962-1981). Belin, Paris

Lefèvre, N., 2007. Le cyclisme d'élite français : un modèle singulier de formation et d'emploi. Thèse de l'université de Nantes.

Le Noé, O., 2000. Socio-histoire des politiques sportives (1940-1975). Genèse d'un groupe de spécialistes de l'administration d'État des activités sportives et structuration du service public du sport. Thèse de l'université Paris I. 
Loirand, G., 1996. Une difficile affaire publique. Une sociologie du contrôle de l'État sur les activités physiques et sportives et sur leur encadrement. Thèse de l'université de Nantes.

Martin, J.-L., 1999. La politique de l'éducation physique sous la $v^{e}$ République. L'élan gaullien (1958-1969). Presses universitaires de France, Paris.

Meimon, J., 2010. Sur le fil. La naissance d'une institution. In: Lagroye, J., Offerlé, M. (Eds), Sociologie de l'institution. Belin, Paris, pp. 105-129.

Noiriel, G., 2006. Introduction à la socio-histoire. La Découverte, Paris.

Offerlé, M., 1994. Sociologie des groupes d'intérêt. Montchrestien, Paris.

Offerlé, M., 1997. Étatisations. Genèses 28, 3-4.

Politix, 2008. Figures de la décision. Politix 82, 3-209 [special issue].

Rasera, F., 2013. Le métier de footballeur. Les coulisses d'une excellence sportive. Thèse de l'université de Lyon 2.

Roger, A., 2003. L'entraînement en athlétisme en France (1919-1973) : une histoire de théoriciens ? Thèse de l'université de Lyon 1.

Sapiro, G., 2006. Les professions intellectuelles entre l'État, l'entrepreneuriat et l'industrie. Le Mouvement social 214, 3-18.

Schotté, M., 2012. La construction du « talent ». La domination des coureurs marocains. Raisons d'agir, Paris.

Turrini, J., 2010. The End of Amateurism in American Track and Field: How the Stars of Track and Field Won Control of Their Sport. University of Illinois Press, Champaign.

Waser, A.-M., 1998. Du monopole fédéral au partage du pouvoir sportif : l'exemple du tennis. Sociétés et représentations 7, 379-394.

Wahl, A., 1990. Le Mai 68 des footballeurs français. Vingtième siècle 26 (1), 73-82.

Wahl, A., Lanfranchi, P., 1995. Les footballeurs français des années trente à nos jours. Hachette, Paris. 